\title{
Heavy-flavour production and the QGP in heavy-ion collisions
}

\section{Roberta Arnaldi*}

INFN Sezione di Torino

E-mail: arnaldiato.infn.it

\begin{abstract}
Heavy-ion collisions are the tool we have to recreate in laboratory the plasma of quarks and gluons. Studies of the QGP formation and properties have been carried on using several probes and now, thirty years after the first measurements at SPS energies, a large wealth of data is available from the high-energy experiments at RHIC and LHC. Heavy-flavour production is considered among the most powerful tools to investigate the new state of matter and the new data can help to sharpen the behaviour of such observables in the created medium. In this paper, an overview of the most recent results on open and closed heavy-flavour will be presented, with particular emphasis on the measurements from RHIC and LHC experiments.
\end{abstract}

EPS-HEP 2017, European Physical Society conference on High Energy Physics 5-12 July 2017

Venice, Italy

${ }^{*}$ Speaker. 


\section{Heavy-ion collisions and quark gluon plasma}

Quantum ChromoDynamics (QCD) predicts, at high temperatures and/or baryonic densities, a phase transition between the hadronic matter and a state, named Quark Gluon Plasma (QGP), where quarks and gluons are no more confined into hadrons. The production and the properties of the QGP can be accessed experimentally by colliding heavy-ions at ultra-relativistic energies. These studies have been performed, for the first time, thirty years ago at CERN SPS, where centreof-mass energies $\left(\sqrt{\mathrm{s}_{\mathrm{NN}}}\right)$ of the order of $17 \mathrm{GeV} / \mathrm{c}$ were reached in $\mathrm{Pb}-\mathrm{Pb}$ interactions. The higher centre-of-mass energies obtained at RHIC $\left(\sqrt{\mathrm{S}_{\mathrm{NN}}}=200 \mathrm{GeV}\right)$ from year 2000 and, ten years later, at LHC (up to $\sqrt{\mathrm{s}_{\mathrm{NN}}}=5.02 \mathrm{TeV}$ ) allows us to explore a system in which even higher temperatures and energy densities are reached. Heavy-flavour particles, being produced in the early stages of the collisions in the initial high- $Q^{2}$ scatterings, probe the entire medium evolution. Since their production is modified by the presence of the QGP, they represent a valuable tool to characterize the created medium.

In heavy-ion facilities, the production of open and closed heavy-flavour are usually studied in three colliding systems, namely in pp, p-A and A-A interactions. The elementary collisions are interesting for QCD studies and they provide a reference for the particle production in $\mathrm{p}$-A or A-A. Proton-nucleus collisions allow the investigation of effects not related to the creation of a hot medium, i.e. the so called "cold nuclear matter" (CNM) effects, as the nuclear modification of parton functions or the initial /final state energy loss of the parton/particle while crossing the medium. Finally, A-A collisions allows the study of the modification induced by the hot QGP medium on the probes. The separation between the expected properties of the three colliding systems is, in reality, not so sharp. Recent results obtained at LHC energies indicate that some behaviours observed in small systems as $\mathrm{pp}$ and $\mathrm{p}-\mathrm{A}$, in particular when high multiplicities are reached, are reminescent of observations done in A-A (e.g. [1, 2]). A new interesting line of research on the role of small systems in heavy-ion physics is now opening up.

A slightly different approach, in structuring the heavy-ion program, have been followed by the RHIC and LHC facilities. At RHIC, the heavy-ion program focussed on the investigation of QGP probes by varying the colliding ions $\left(\mathrm{Cu}, \mathrm{Au}\right.$ or $\mathrm{U}$ ) and the energy of the collisions (from $\sqrt{\mathrm{s}_{\mathrm{NN}}}=$ $39 \mathrm{GeV} / \mathrm{c}$ to $200 \mathrm{GeV} / \mathrm{c}$. At LHC, the peculiarity is not only the fact that top A-A collision energies are reached $\left(\sqrt{\mathrm{s}_{\mathrm{NN}}}=5.02 \mathrm{TeV}\right.$ for $\mathrm{Pb}-\mathrm{Pb}$ and $\sqrt{\mathrm{S}_{\mathrm{NN}}}=8.16 \mathrm{TeV}$ for $\left.\mathrm{p}-\mathrm{Pb}\right)$, but also the fact that all of the four main LHC experiments (ALICE, ATLAS, LHCb and CMS) take part to the heavy-ion program. These experiments, being characterised by very different, but complementary, kinematic coverages, provide an unique way to investigate heavy-flavour probes over a very broad transverse momentum $\left(p_{\mathrm{T}}\right)$ and rapidity $(y)$ range.

All the experiments both at RHIC and LHC usually evaluate the modification of the heavyflavour production yields, induced by either hot or cold matter effects, through the nuclear modification factor $\left(R_{\mathrm{AA}}\right) . R_{\mathrm{AA}}$ is defined as the ratio of the particle yields in A-A or p-A collisions $\left(Y_{\mathrm{AA}}\right)$ and the expected value obtained by scaling the pp yield $\left(Y_{\mathrm{pp}}\right)$ by the average number of nucleon-nucleon collisions, $\left\langle N_{\text {coll }}\right\rangle$, evaluated through a Glauber model calculation:

$$
R_{\mathrm{AA}}=Y_{\mathrm{AA}} /\left(\left\langle N_{\text {coll }}\right\rangle \times Y_{\mathrm{pp}}\right)
$$

$R_{\mathrm{AA}}$ is expected to be equal to unity if the particle yield in A-A scales with $N_{\text {coll }}$, as it is the 
case for electroweak probes (direct $\gamma, \mathrm{W}, \mathrm{Z}$ ) which do not interact strongly. On the contrary, $R_{\mathrm{AA}}$ different from unity might imply that the particle production is affected by the medium, through hot or cold matter effects. Furthermore, in A-A collisions, the modification induced by the created medium is expected to be more important in head-on collisions. The centrality of the interaction, usually obtained from the measurement of the particle multiplicity or the energy released at forward rapidity through the Glauber model calculation, is therefore a crucial parameter to classify the events for heavy-flavour studies.

\section{Open heavy-flavours}

Heavy-flavours are produced in high- $Q^{2}$ hard-scattering, in the early collision stages. Therefore, they probe the entire medium evolution in their path out of the colliding zone. They interact strongly with the constituents of the medium, through parton energy loss processes (either of radiative or collisional origin) and through the partecipation to the collective flow. The amount of lost energy is influenced by several factors. First it depends on the path length of the particle inside the medium and on the density of the medium itself. Secondly, the energy loss is influenced by the color charge, being more important for gluons than for quarks, and by the mass of the quark $\left(m_{q}\right)$, since the emission of the gluon radiation is expected to be suppressed in a cone of aperture angle proportional to $m_{q}$. In this scenario, a hierarchy in the amount of energy which is lost is expected, being larger for open charm and smaller for the heavier open beauty mesons.

In Fig. 1 (left), the nuclear modification factor of D mesons is shown, as a function of the transverse momentum [3]. A clear suppression, stronger in most central collisions, can be observed. Such a suppression is similar in size at both $\sqrt{\mathrm{S}_{\mathrm{NN}}}=2.76 \mathrm{TeV}$ and $5.02 \mathrm{TeV}$. This is expected from theory models combining the effect of the higher temperature reached at $\sqrt{\mathrm{s}_{\mathrm{NN}}}=5.02 \mathrm{TeV}$, which would decrease the $R_{\mathrm{AA}}$ value, with the harder $p_{\mathrm{T}}$ distribution of charm quarks at $\sqrt{\mathrm{s}_{\mathrm{NN}}}=5.02$ $\mathrm{TeV}$, which would, on the contrary, increase the $R_{\mathrm{AA}}[4]$.
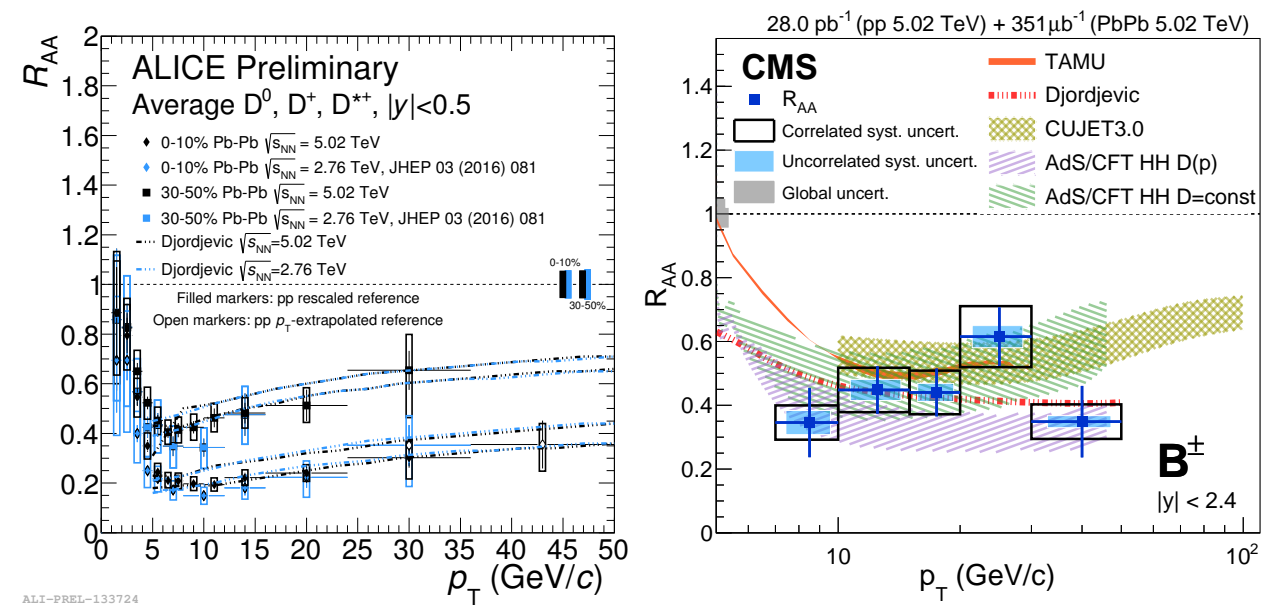

Figure 1: Left: ALICE $R_{\mathrm{AA}}$ measurement for D mesons as a function of $p_{\mathrm{T}}$ at $\sqrt{\mathrm{S}_{\mathrm{NN}}}=5.02$ and $2.76 \mathrm{TeV}$ [3], compared with theory curves [4]. Right: B mesons $R_{\mathrm{AA}}$ [5], measured by CMS, shown as a function of $p_{\mathrm{T}}$ [5]

The nuclear modification factor of B mesons is obtained either studying non-prompt $\mathrm{J} / \psi[6]$ or by reconstructing the $B \rightarrow J / \psi K^{ \pm}$decay [5]. A strong suppression, almost $p_{\mathrm{T}}$-independent can be 
observed in both cases. Models including different descriptions of the $\mathrm{Pb}-\mathrm{Pb}$ medium and different energy loss sources reasonably describe the data, but it is not possible yet to disentangle among the various approaches, given the current statistical and systematic uncertainties. When the $R_{\mathrm{AA}}$ of open charm and open beauty mesons are compared, their behaviours differ depending on the $p_{\mathrm{T}}$ region under study. At low- $p_{\mathrm{T}}$ (Fig. 2 left), the $R_{\mathrm{AA}}$ of the $\mathrm{B}$ mesons (from non-prompt $\mathrm{J} / \psi$ [7]) is larger, over all centralities, than the one of the D mesons [8], confirming the aforementioned mass-dependent energy loss. At high $p_{\mathrm{T}}\left(p_{\mathrm{T}}>10 \mathrm{GeV} / \mathrm{c}\right)$, the flavour dependence tends to vanish and the $R_{\mathrm{AA}}$ of B and D mesons become similar and close to the one of light hadrons $[9,5]$.
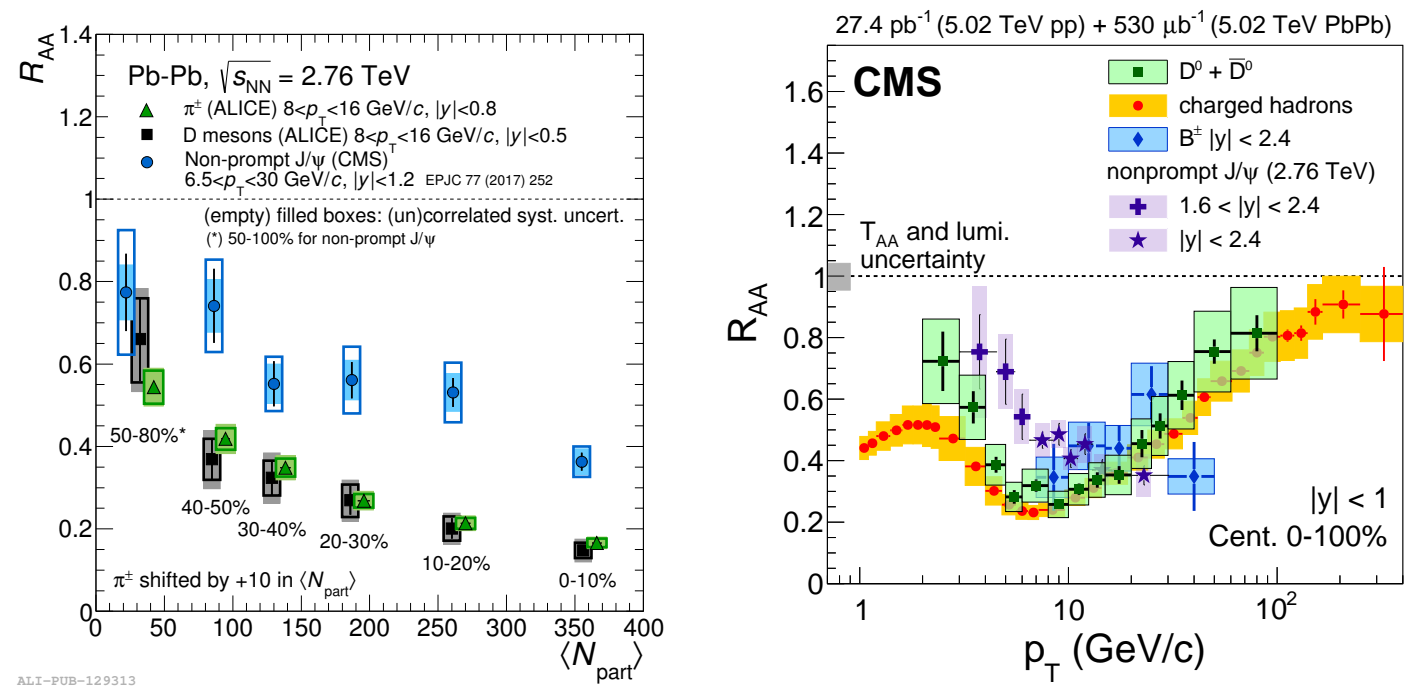

Figure 2: Comparison of the $\mathrm{D}$ and $\mathrm{B}$ mesons $R_{\mathrm{AA}}$ at low $p_{\mathrm{T}}$ (left) and high $p_{\mathrm{T}}$ (right)

Further evidence of the interactions of charm quarks with the medium is provided by the observation of their elliptic flow, which measures the size of the collective flow imparted by the medium to the charm. The collective dynamics of the medium, in fact, converts, during its expansion, the initial spatial anisotropy into a final state particle momentum anisotropy. The coefficients of the Fourier expansion of the azimuthal angle distribution, with respect to the event plane, characterize this momentum anisotropy and the second coefficient, $v_{2}$, corresponds to the so-called elliptic flow. A positive elliptic flow was already observed at RHIC. The LHC results confirm the observation (Fig. 3, right), with a higher accuracy [10]. The comparison of the D meson $v_{2}$ to the one of the pions and with model calculations indicates that the low-momentum charm quarks take part to the collective QGP motion. The comparison with theory models suggests a thermalization time around 3-14 fm/c, representing a first step towards the characterization of the QGP with heavy quarks.

\section{Quarkonium}

Quarkonium is a bound state of a $Q$ and a $\bar{Q}$ pair, where $Q$ can be either a charm quark or a bottom quark, forming respectively a charmonium or a bottomonium state. In a hot and deconfined medium, the production of quarkonium is expected to be significantly suppressed with respect to the pp yield scaled by the number of nucleon-nucleon collisions. The mechanism driving this suppression is the color screening of the force which binds the $c \bar{c}(b \bar{b})$ state [11]. In this scenario, 
quarkonium suppression should happen in a sequential way, according to the binding energy of the various resonances. In the quarkonium sector, strongly bound states as the $\mathrm{J} / \psi$ should melt at higher temperatures with respect to the more loosely bound $\chi_{c}$ and $\psi(2 \mathrm{~S})$ states. Similarly, in the bottomonium sector, the $\Upsilon(1 \mathrm{~S})$ will melt at higher temperatures with respect to the $\Upsilon(2 \mathrm{~S})$ and $\Upsilon(3 S)$. As a consequence, the in-medium dissociation probability of all these states should reflect the temperature reached by the system [12]. However, the link between the dissociation temperature and the disapperance of a given resonance is not yet settled in an univocal way. On the theory side, large uncertainties are associated to the definition of such a temperature, depending on the adopted approach [13]. Experimentally, several competing mechanisms can affect the sequential suppression pattern. The feed-down contributions from higher-masses resonances and the B-hadron decay into charmonium should, for example, be considered. Furthermore, other cold and hot matter effects can compete with the suppression mechanisms. Cold nuclear matter effects, as the modification of the parton distributions into nuclei (known as shadowing), the formation of a color glass condensate or energy loss processes can influence the quarkonium production. Increasing the centre-of-mass energy of nucleus-nucleus collisions, an increase in the production of $Q$ and $\bar{Q}$ quarks is also expected. Therefore, at high $\sqrt{\mathrm{s}_{\mathrm{NN}}}$, the abundance of $Q$ and $\bar{Q}$ quarks may lead to (re)combination processes, either at the hadronization $[14,15]$ or during the collision history [16], which can counterbalance, partially or totally, the effect of the suppression mechanisms. (Re)combination is more important for charmonium states than for the bottomonium ones, given the higher number of $c \bar{c}$ with respect to $b \bar{b}$ pairs.

The first quarkonium measurements in heavy-ion collisions, performed at the CERN SPS [17, 18], indicate a clear suppression of the $\mathrm{J} / \psi$ yield, well beyond the expectations based just on cold nuclear matter effects (the so-called "anomalous $J / \psi$ suppression"). Results obtained at RHIC indicate a $\mathrm{J} / \psi$ suppression similar to the one observed at SPS, in spite of the factor ten increase in $\sqrt{\mathrm{S}_{\mathrm{NN}}}[19,20]$. Unexpectedly, a stronger $\mathrm{J} / \psi$ suppression was observed at forward with respect to mid-rapidity, although the larger energy density reached at $y \sim 0$. These observations can be framed in a scenario which includes the production of charmonia through (re)combination mechanisms, more important at higher $\sqrt{\mathrm{S}_{\mathrm{NN}}}$ and playing a role in particular at mid-rapidity, where the density of charm quarks is higher. At LHC, quarkonium studies have been carried on over a broad kinematic coverage, provided by the complementarity of the four main experiments. The highest energies reached at LHC create an ideal situation for testing the suppression and (re)combination scenarios, which are both expected to be stronger at high $\sqrt{\mathrm{S}_{\mathrm{NN}}}$, due to the increase of the energy density reached in the collision and the increase in the charm quarks production cross section.

The centrality dependence of the $\mathrm{J} / \psi$ nuclear modification factor is shown in Fig. 3 (left), where the ALICE measurements at $\sqrt{\mathrm{s}_{\mathrm{NN}}}=2.76$ and $5.02 \mathrm{TeV}[21,22]$ are compared to the $R_{\mathrm{AA}}$ measured, at RHIC energies, by PHENIX [20]. In both cases, the kinematic range is characterized by the coverage of the low $p_{\mathrm{T}}$ region. There is a clear evidence for a smaller $\mathrm{J} / \psi$ suppression at LHC with respect to RHIC, with a hint for a slightly stronger suppression at $\sqrt{\mathrm{s}_{\mathrm{NN}}}=2.76$ $\mathrm{TeV}$ with respect to $\sqrt{\mathrm{s}_{\mathrm{NN}}}=5.02 \mathrm{TeV}$. Furthermore, while PHENIX results shown a suppression which increases with centrality, the LHC ones saturate for $N_{\text {part }}>100$. Transport models, including suppression and (re)combination contributions, predict such a behaviour [24, 25]. A similar trend is expected also by the statistical model [15], where the $\mathrm{J} / \psi$ yield is completely determinated by the abundance of $c \bar{c}$ pairs and by the chemical freeze-out conditions. The comparison between data 

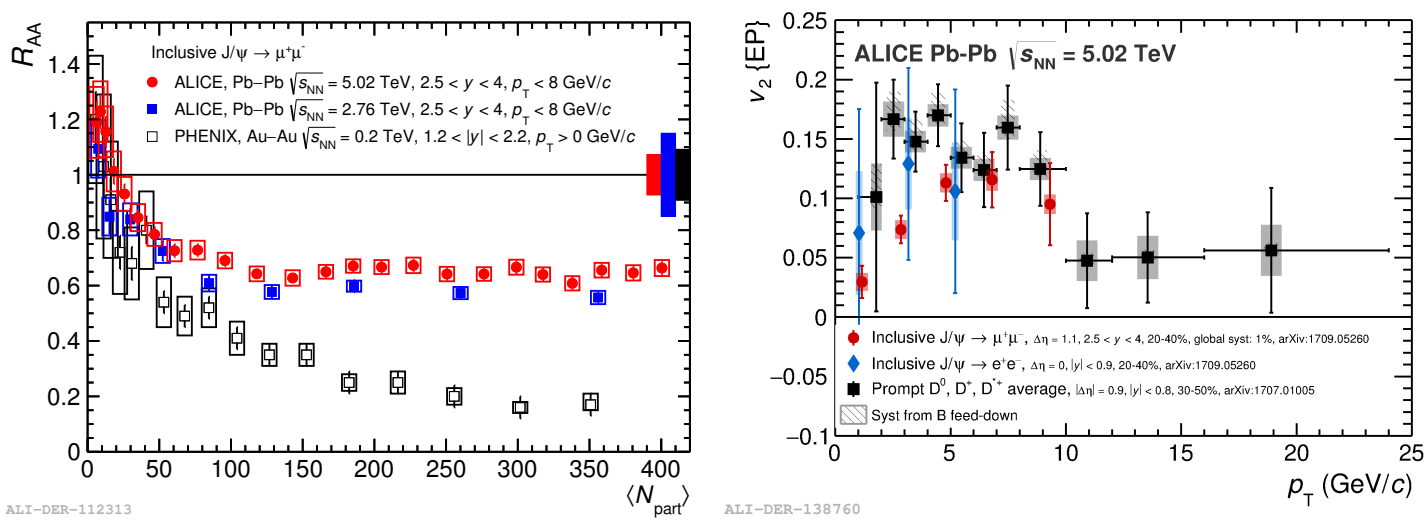

Figure 3: Left: ALICE [21, 22] and PHENIX [20] low $p_{\mathrm{T}} \mathrm{J} / \psi R_{\mathrm{AA}}$ versus the number of participant nucleons at forward rapidity. Right: $\mathrm{J} / \psi$ and $\mathrm{D} v_{2}$ as a function of the transverse momentum [23, 10]

and theory models will clearly benefit by an experimental measurement of the charm production cross section, which represents one of the largest uncertainties so far. The (re)combination process dominates in central collisions and, for kinematical reasons, at low $p_{\mathrm{T}}$, becoming negligible as the $\mathrm{J} / \psi p_{\mathrm{T}}$ increases. This is confirmed by the results obtained by CMS [26] and STAR [27] for high $p_{\mathrm{T}} \mathrm{J} / \psi$, i.e. for $\mathrm{J} / \psi$ measured in a kinematic region where the (re)combination contribution can be neglected. In this case, the observed suppression is stronger at LHC energies, as expected in a dissociation scenario.

The $R_{\mathrm{AA}}$ study of the $\mathrm{J} / \psi$ is complemented by the measurement of the elliptic flow $v_{2}$. The ALICE result for $\mathrm{Pb}-\mathrm{Pb}$ collisions at $\sqrt{\mathrm{S}_{\mathrm{NN}}}=5.02 \mathrm{TeV}$ shows that the $\mathrm{J} / \psi v_{2}$ is significantly different from zero $(\sim 7 \sigma$ effect $)$ at intermediate $p_{\mathrm{T}}$ [23]. This indicates that the $\mathrm{J} / \psi$ flows, as expected in a (re)combination scenario in which the $\mathrm{J} / \psi$ inherits the flow of the charm quark. The comparison of the D [10] and J/ $\psi$ flow, which turns out to be rather similar, as visible in Fig. 3 (right), can provide insight on the interactions of heavy and light quarks in the medium.
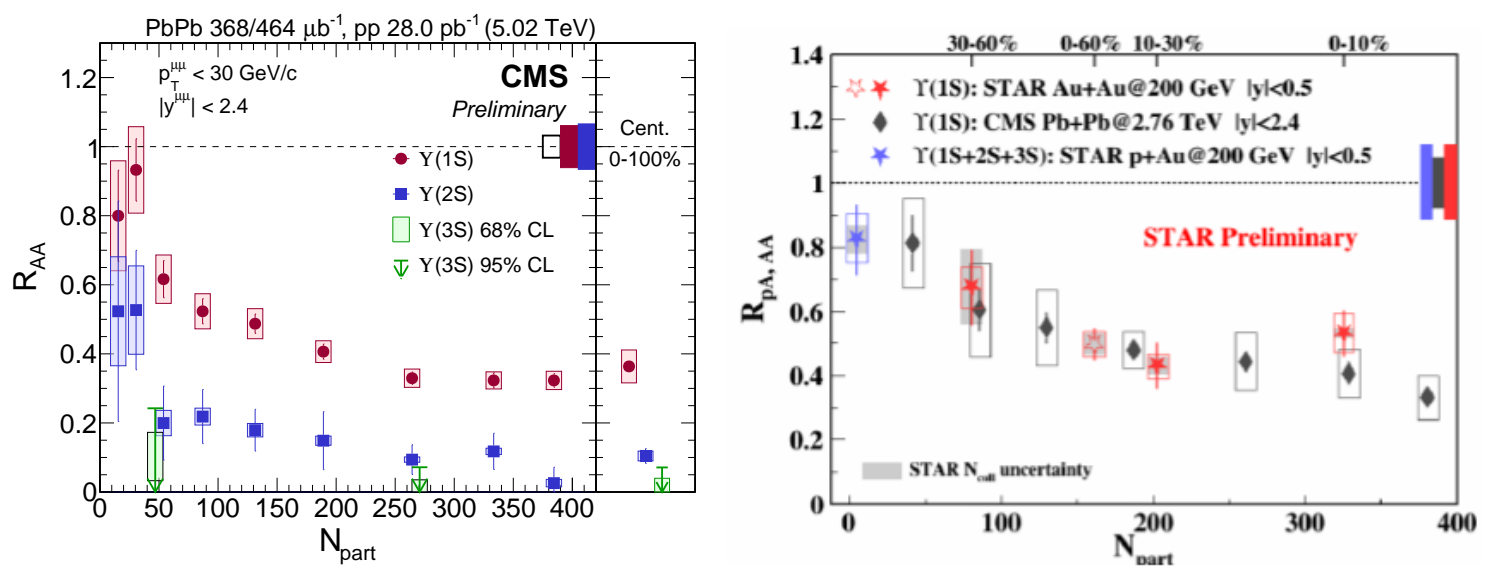

Figure 4: $\Upsilon R_{\mathrm{AA}}$ versus centrality, measured by CMS[28](left) and by STAR (right)

Finally, the high energies reached at LHC allow a precise study of the bottomonium family. 
CMS results at $\sqrt{\mathrm{s}_{\mathrm{NN}}}=5.02 \mathrm{TeV}$ [28] confirm the $\Upsilon R_{\mathrm{AA}}$ measurement at $\sqrt{\mathrm{S}_{\mathrm{NN}}}=2.76 \mathrm{TeV}$ [29]. Given the low expected level of (re)combination, the observed pattern, shown in Fig. 4 (left), can be interpreted as the sequential suppression of $\Upsilon(1 S), \Upsilon(2 S)$ and $\Upsilon(3 S)$. A similar $\Upsilon(1 S) R_{\mathrm{AA}}$ is also observed in recent STAR results. To understand if the temperature reached in the medium are indeed high enough to suppress also the most strongly bound resonance of the quarkonium family, precise measurements of feed-down from excited states and of cold nuclear matter effects at RHIC and LHC energies are needed. A precise knowledge of cold matter effects has, in fact, to be achieved to quantify the size of hot matter effects. This is feasible through the study of p-A (or d-A) collisions. Recent results from ALICE [30, 31] and LHCb [32] at top p-A collisions energy $\left(\sqrt{\mathrm{S}_{\mathrm{NN}}}=8.16 \mathrm{TeV}\right)$ show that the $\mathrm{J} / \psi$ production yield is strongly modified as a function of rapidity and the modification is stronger in the most central collisions, as it can be seen in Fig. 5. The results confirm, with a higher precision, those already obtained at $\sqrt{\mathrm{s}_{\mathrm{NN}}}=5.02 \mathrm{TeV}[33,34]$. As shown in Fig. 5 (left), the $y$ dependence of the $\mathrm{J} / \psi$ nuclear modification factor is in agreement with theory models based on a pure cold nuclear matter effects as nuclear shadowing, as well as partonic energy loss or color glass condensate.
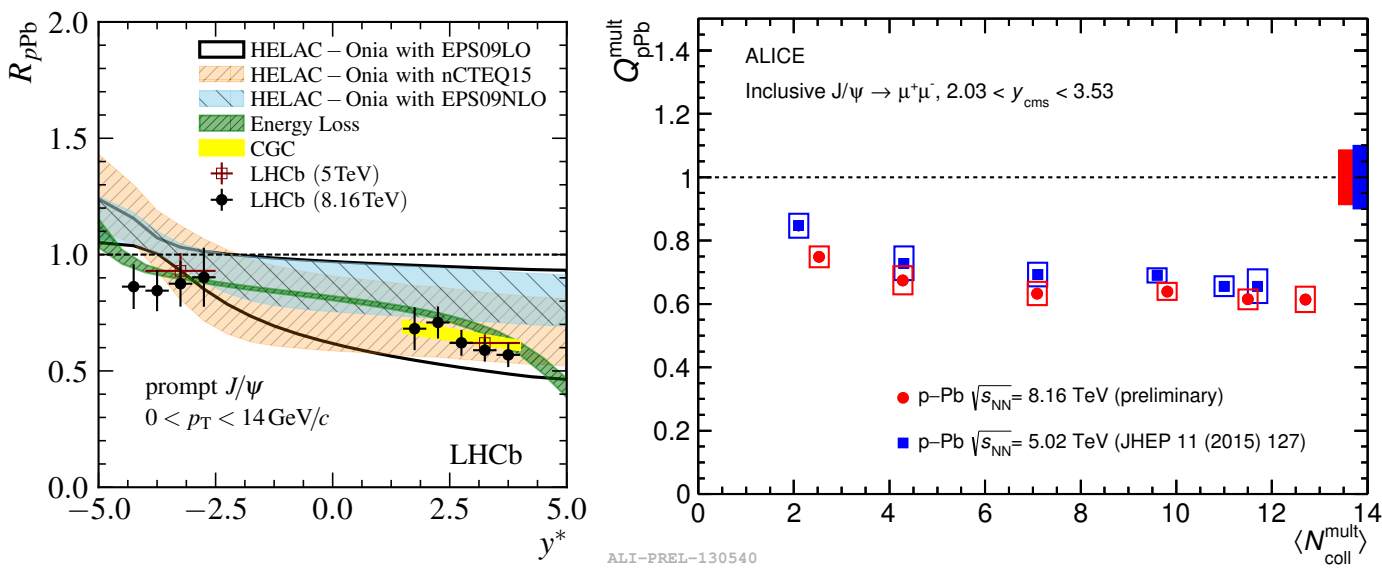

Figure 5: Left: $R_{\mathrm{AA}}$ measured by LHCb at $\sqrt{\mathrm{s}_{\mathrm{NN}}}=8.16 \mathrm{TeV}$ compared to several theory calculations. Right: nuclear modification factor $\left(Q_{P P b}\right)$ measured by ALICE as a function of centrality at forward rapidity [31]

Similar studies in p-A collisions have been performed also for the $\Upsilon$ states. In the rather large rapidity range covered by the four LHC experiments, no clear rapidity dependence is observed and the size of cold nuclear matter effects is weaker with respect to the $\mathrm{J} / \psi$.

\section{Conclusions}

Open and closed heavy-flavour are confirmed to be crucial tools to investigate different aspects of the plasma of quarks and gluons. The most recent results from RHIC and LHC have remarkably increased the precision reached so far, allowing a very detailed study of both the nuclear modification factor and the elliptic flow. A simultaneous description of those two aspects of the interaction between particles and medium is now challenging for theoretical models. Futhermore, the accuracy of the new results allow comparative studies of B and D mesons, in the open heavy-flavour sector, and charmonia and bottomonia in the closed heavy-flavour one. These measurements, now within 
reach in a wide kinematic range and at several centre-of-mass energies, are going to sharpen our knowledge, providing further insight in the characterization of the QGP.

\section{References}

[1] CMS Collaboration, V. Khachatryan et al., "Observation of Long-Range Near-Side Angular Correlations in pp Collisions at the LHC," JHEP 09 (2010) 091, arXiv:1009.4122 [hep-ex].

[2] CMS Collaboration, S. Chatrchyan et al., "Observation of long-range near-side angular correlations in pPb collisions at the LHC," Phys. Lett. B718 (2013) 795-814, arXiv:1210.5482 [nucl-ex].

[3] ALICE Collaboration, "Preliminary Physics Summary: Measurement of $D^{0}, D^{+}, D^{*}$ and $D_{s}^{+}$ production in $\mathrm{Pb}-\mathrm{Pb}$ collisions at $\sqrt{s_{N N}}=5.02 \mathrm{TeV}$." Alice-public-2017-003, 2017.

[4] M. Djordjevic and M. Djordjevic, "Predictions of heavy-flavor suppression at $5.1 \mathrm{TeV} \mathrm{Pb}+\mathrm{Pb}$ collisions at the CERN Large Hadron Collider,” Phys. Rev. C92 no. 2, (2015) 024918, arXiv:1505.04316 [nucl-th].

[5] CMS Collaboration, A. M. Sirunyan et al., "Measurement of the $B^{ \pm}$Meson Nuclear Modification Factor in $\mathrm{Pb}-\mathrm{Pb}$ Collisions at $\sqrt{s_{N N}}=5.02 \mathrm{TeV}$," Phys. Rev. Lett. 119 no. 15, (2017) 152301, arXiv:1705.04727 [hep-ex].

[6] ATLAS Collaboration, "Study of $J / \psi$ and $\psi(2 S)$ production with $2015 \mathrm{PbPb}$ data at $\sqrt{s_{N N}}=5.02$ $\mathrm{TeV}$ and pp data at $\sqrt{s}=5.02 \mathrm{TeV}$ with the ATLAS detector." Atlas-conf-2016-109, 2016.

[7] CMS Collaboration, V. Khachatryan et al., "Suppression and azimuthal anisotropy of prompt and nonprompt $\mathrm{J} / \psi$ production in $\mathrm{PbPb}$ collisions at $\sqrt{s_{N N}}=2.76 \mathrm{TeV}$," Eur. Phys. J. C77 no. 4, (2017) 252, arXiv:1610.00613 [nucl-ex].

[8] ALICE Collaboration, J. Adam et al., "Centrality dependence of high- $\mathrm{p}_{T} \mathrm{D}$ meson suppression in $\mathrm{Pb}-\mathrm{Pb}$ collisions at $\sqrt{s_{\mathrm{NN}}}=2.76 \mathrm{TeV}$, JHEP 11 (2015) 205, arXiv:1506.06604 [nucl-ex] . [Addendum: JHEP06,032(2017)].

[9] CMS Collaboration, A. M. Sirunyan et al., "Nuclear modification factor of D0 mesons in $\mathrm{PbPb}$ collisions at sqrt(s[NN]) =5.02 TeV," arXiv:1708.04962 [nucl-ex].

[10] ALICE Collaboration, S. Acharya et al., "D-meson azimuthal anisotropy in mid-central Pb-Pb collisions at $\sqrt{\mathbf{s}_{\mathrm{NN}}}=\mathbf{5 . 0 2} \mathrm{TeV}, " \operatorname{arXiv}: 1707.01005$ [nucl-ex].

[11] T. Matsui and H. Satz, “J/ $\psi$ Suppression by QGP Formation,” Phys. Lett. B178 (1986) 416-422.

[12] S. Digal, P. Petreczky, and H. Satz, “Quarkonium feed down and sequential suppression,” Phys. Rev. D64 (2001) 094015, arXiv: hep-ph/0106017 [hep-ph].

[13] PHENIX Collaboration, A. Adare et al., "Measurement of $\Upsilon(1 S+2 S+3 S)$ production in $p+p$ and $\mathrm{Au}+\mathrm{Au}$ collisions at $\sqrt{s_{N N}}=200 \mathrm{GeV}$," Phys. Rev. C91 no. 2, (2015) 024913, arXiv:1404.2246 [nucl-ex].

[14] P. Braun-Munzinger and J. Stachel, “(Non)thermal aspects of charmonium production and a new look at J/ $\psi$ suppression,” Phys. Lett. B490 (2000) 196-202, arXiv:nucl-th/0007059 [nucl-th].

[15] A. Andronic, P. Braun-Munzinger, K. Redlich, and J. Stachel, "The thermal model on the verge of the ultimate test: particle production in Pb-Pb collisions at the LHC," J. Phys. G38 (2011) 124081, arXiv:1106.6321 [nucl-th].

[16] R. L. Thews, M. Schroedter, and J. Rafelski, "Enhanced $J / \psi$ production in deconfined quark matter," Phys. Rev. C63 (2001) 054905, arXiv: hep-ph/0007323 [hep-ph]. 
[17] NA50 Collaboration, M. C. Abreu et al., "Evidence for deconfinement of quarks and gluons from the $\mathrm{J} / \psi$ suppression pattern measured in PbPb collisions at CERN SPS," Phys. Lett. B477 (2000) 28-36.

[18] NA60 Collaboration, R. Arnaldi et al., "J/ $\psi$ production in In-In collisions at 158-GeV/nucleon," Phys. Rev. Lett. 99 (2007) 132302.

[19] PHENIX Collaboration, A. Adare et al., " $J / \psi$ Production vs Centrality, $p_{T}$, and $y$ in Au+Au at $\sqrt{s_{N N}}=200 \mathrm{GeV}$, Phys. Rev. Lett. 98 (2007) 232301, arXiv:nucl-ex/0611020 [nucl-ex].

[20] PHENIX Collaboration, A. Adare et al., " $J / \psi$ suppression at forward rapidity in Au+Au collisions at $\sqrt{s_{N N}}=200 \mathrm{GeV}, "$ Phys. Rev. C84 (2011) 054912, arXiv:1103.6269 [nucl-ex].

[21] ALICE Collaboration, B. B. Abelev et al., "Centrality, rapidity and transverse momentum dependence of $J / \psi$ suppression in Pb-Pb collisions at $\sqrt{s_{\mathrm{NN}}}=2.76 \mathrm{TeV}$," Phys. Lett. B734 (2014) 314-327, arXiv:1311.0214 [nucl-ex].

[22] ALICE Collaboration, J. Adam et al., "J/ $\psi$ suppression at forward rapidity in $\mathrm{Pb}-\mathrm{Pb}$ collisions at $\sqrt{\mathbf{s}_{\mathrm{NN}}}=\mathbf{5 . 0 2} \mathrm{TeV}$, , Phys. Lett. B766 (2017) 212-224, arXiv:1606.08197 [nucl-ex] .

[23] ALICE Collaboration, S. Acharya et al., "J/ $\psi$ elliptic flow in Pb-Pb collisions at $\sqrt{\mathbf{s}_{\mathrm{NN}}}=5.02 \mathrm{TeV}$," arXiv:1709.05260 [nucl-ex].

[24] X. Zhao and R. Rapp, "Medium Modifications and Production of Charmonia at LHC," Nucl. Phys. A859 (2011) 114-125, arXiv:1102.2194 [hep-ph] .

[25] Y.-p. Liu, Z. Qu, N. Xu, and P.-f. Zhuang, “J/ $\psi$ Transverse Momentum Distribution in High Energy Nuclear Collisions at RHIC,” Phys. Lett. B678 (2009) 72-76, arXiv:0901.2757 [nucl-th].

[26] CMS Collaboration, "Measurement of prompt and nonprompt charmonium suppression in $\mathrm{PbPb}$ collisions at 5.02 TeV." Cms-pas-hin-16-025, 2017.

[27] STAR Collaboration, L. Adamczyk et al., " $J / \psi$ production at high $p_{T}$ in $p+p$ and Au+Au collisions at $\sqrt{s_{N N}}=200 \mathrm{GeV}$," Phys. Lett. B722 (2013) 55-62, arXiv:1208.2736 [nucl-ex].

[28] CMS Collaboration, A. M. Sirunyan et al., "Suppression of excited Upsilon states relative to the ground state in $\mathrm{PbPb}$ collisions at $\operatorname{sqrt}(\mathrm{sNN})=5.02 \mathrm{TeV}$," arXiv:1706.05984 [hep-ex] .

[29] CMS Collaboration, V. Khachatryan et al., "Suppression of $\Upsilon(1 S), \Upsilon(2 S), \Upsilon(3 S)$ production in $\mathrm{PbPb}$ collisions at $\sqrt{s_{\mathrm{NN}}}=2.76 \mathrm{TeV}$, , Phys. Lett. B770 (2017) 357-379, arXiv: 1611.01510 [nucl-ex].

[30] ALICE Collaboration, "Preliminary Physics Summary: Inclusive J $\psi$ production at forward rapidity in p-Pb collisions at $\sqrt{s_{N N}}=8.16$ TeV." Alice-public-2017-001, 2017.

[31] ALICE Collaboration, "Preliminary Physics Summary: Centrality dependence of inclusive J $\psi$ production in $\mathrm{Pb}-\mathrm{Pb}$ collisions at $\sqrt{s_{N N}}=8.16 \mathrm{TeV}$." Alice-public-2017-007, 2017.

[32] LHCb Collaboration, R. Aaij et al., "Prompt and nonprompt $\mathrm{J} / \psi$ production and nuclear modification in $p \mathrm{~Pb}$ collisions at $\sqrt{s_{N N}}=8.16 \mathrm{TeV}$, "Phys. Lett. B774 (2017) 159-178, arXiv: 1706.07122 [hep-ex].

[33] ALICE Collaboration, J. Adam et al., "Rapidity and transverse-momentum dependence of the inclusive $\mathrm{J} / \psi$ nuclear modification factor in p-Pb collisions at $\sqrt{s_{N N}}=5.02 \mathrm{TeV}$, , JHEP 06 (2015) 055, arXiv:1503.07179 [nucl-ex].

[34] ALICE Collaboration, J. Adam et al., "Centrality dependence of $\psi(2 \mathrm{~S})$ suppression in $\mathrm{p}-\mathrm{Pb}$ collisions at $\sqrt{s_{\mathrm{NN}}}=5.02 \mathrm{TeV}$," JHEP 06 (2016) 050, arXiv:1603.02816 [nucl-ex] . 